\title{
The Oscillation of the Nonlinear Functional Equation with Variable Coefficients
}

\author{
Quanwen Lin, Yingzhu Wu \& Siquan Liao \\ Department of Mathematics, Maoming university, Maoming 525000, China \\ E-mail: linquwen@126.com
}

Foundation item: Supported by the NSF of Maoming university.

\section{Abstract}

By studying the nonlinear functional equation with variable coefficients

$$
x(g(t))=P(t) x(t)+Q(t) \prod_{i=1}^{m}\left|x\left(g^{k_{i}+M}(t)\right)\right|^{\alpha_{j}} \text { sign } x\left(g^{k_{i}+M}(t)\right)
$$

the oscillation of the solution in this paper, we reached some new oscillation criterion. These criterion will expand or improved some results of [1],[2],[4] and [6].

Keywords: Nonlinear, Variable coefficient, Functional equations, Oscillation criterion

\section{Introduction}

Considering the nonlinear functional equation with variable coefficients:

$$
x(g(t))=P(t) x(t)+Q(t) \prod_{i=1}^{m}\left|x\left(g^{k_{i}+M}(t)\right)\right|^{\alpha_{i}} \operatorname{sign} x\left(g^{k_{i}+i}(t)\right),
$$

where $k_{i} \geqslant 1, M \geqslant 1$ is a positive integer, $\alpha_{i}$ is not a positive real number, $i=1,2,3 \cdots m$ and $\sum_{i=1}^{m} \alpha_{i}=1 ; P(t), Q(t): I \rightarrow$ $R^{+}=(0,+\infty)$ are given real valued functions, and I denotes an unbonunded subset of $R^{+} . x$ is an unknown real valued function, $g(t): I \rightarrow I$ are given functions $\lim g(t)=\infty, t \in I$ and $g^{i}$ denotes the ith iterate of the function g.i.e.

$$
g^{0}(t)=t, g^{i+1}(t)=g\left(g^{i}(t)\right), t \in I, i=1,2, \cdots
$$

1n 1994, Golda and Werbowski [1] had studied the second linear functional equation of the form:

$$
x(g(t))=p(t) x(t)+Q(t) x\left(g^{2}(t)\right), t \geqslant t_{0}
$$

They proved:

Theorem 1.1 All the solution of the functional equation (1.2) is oscillation, if

$$
\liminf _{t \rightarrow \infty} Q(t) P(g(t))>\frac{1}{4}
$$

or

$$
\limsup _{t \rightarrow \infty} Q(t) P(g(t))>1
$$

By the year of 1995, Nowakowska and Werbowski [2] expanded the condition (1.3) to the higher order linear functional equation:

$$
x(g(t))=P(x) x(t)+\sum_{i=1}^{k} Q_{i}(t) x\left(g^{i+1}(t)\right)
$$


In this condition, they reached

Theorem 1.2 All the sulutions of the equation oscillate, if

$$
\liminf _{t \rightarrow \infty} \sum_{i=1}^{K} Q_{i}(t) \prod_{j=1}^{K} P\left(g^{j}(t)\right)>\frac{1}{4}
$$

or

$$
\limsup _{t \rightarrow \infty} \sum_{i=0}^{K-1} G\left(g^{i}(t)\right) \prod_{j=1}^{K} P\left(g^{i+j}(t)\right)>\left(\frac{K}{K+1}\right)^{K+1}
$$

and

$$
G(t)=\sum_{n=1}^{K-1} Q_{n}(t) Q_{K-n}\left(g^{n}(t)\right)++Q_{K}(t)
$$

Golda and Werbowski [1] had pointed out that if the condition (1.3) was inexistent, the functional equation had nonoscillatory solutions. So, when the conditions (1.3) and (1.4) is non-existent, how to set up the oscillation condition of the functional equation (1.2) and how to fill up the bland between the conditions (1.3) and (1.4) are questions worth studying. By the year of 1999, the scholars of China Zhou Yong and Yu Yuan-hong had studied the oscillation solution of the equation and answered the questions above:

$$
x(g(t))=P(x) x(t)+\sum_{i=1}^{m} Q_{i}(t) x\left(g^{K+i}(t)\right)
$$

they proved

Theorem 1.3 All the solutions of the functional equation (1.9) oscillate if

$$
\liminf _{t \rightarrow \infty} \sum_{i=1}^{m} Q_{i}(t) \prod_{j=1}^{K+i-1} P\left(g^{j}(t)\right)=A>\frac{K^{K}}{(K+1)^{K+1}}
$$

or

$$
0 \leq A \leq \frac{K^{k}}{(K+1)^{K+1}}, \limsup _{t \rightarrow \infty} \sum_{i=1}^{m} Q_{i}(t) \prod_{j=1}^{K+i-1} P\left(g^{j}(t)\right)>\frac{1}{[\lambda(A)]^{K}}
$$

Here, $\lambda(A)$ is the only real roof of equation

$$
A \lambda^{K+1}-\lambda+1=0
$$

in $\left[1,((K+1) A)^{-\frac{1}{K}}\right]$, and reached (when $\left.m=1, K=1\right)$.

Corollary 1.1 All the solutions of functional equation (1.2) oscillate if

$$
\liminf _{t \rightarrow \infty} Q(t) P(g(t))=A>\frac{1}{4}
$$

or

$$
0 \leq A \leq \frac{1}{4}, \limsup _{t \rightarrow \infty} Q(t) P(g(t))=>\frac{1+\sqrt{1-4 A}}{2}
$$

Obviously, the condition (1.14) had improved the condition (1.4).

By the year of 2000, Zhou Yong, Liu Zheng-rong and Yu Yuan-hong had set up a class of the higher nonlinear functional equation :

$$
x(g(t))=P(t) x(t)+Q(t) \prod_{i=1}^{m}\left|x\left(g^{k_{i}+1}(t)\right)\right|^{\alpha_{i}} \operatorname{sign} x\left(g^{k_{i}+1}(t)\right),
$$

and $k_{i} \geq 1$ is a positive integer, $\alpha_{i}(i=1,2, \cdots m)$ is not a passitive real number and $\sum_{i=1}^{m} \alpha_{i}=1$,Equation: $P, Q: I \rightarrow R^{+}=$ $(0, \infty), I$ are given real valued functions $\infty \geq 0 \mathrm{x}$ is an unknown real valued function, $g(t) \neq t$ for $t \geq 0, \lim _{t \rightarrow \infty} g(t)=\infty$. Mark down $K=\sum_{i=1}^{m} \alpha_{i} k_{i}$ They had proved.

Theorem 1.4 Supposed

$$
\liminf _{t \rightarrow \infty} Q(t) \prod_{i=1}^{m}\left[\prod_{j=1}^{k_{i}} P\left(g^{j}(t)\right)\right]^{\alpha_{i}}=A>\frac{K^{K}}{(K+1)^{K+1}}
$$


then all the solutions of the functional equation (1.15) oscillated. and

Theorem 1.5 Supposed

$$
\liminf _{t \rightarrow \infty} Q(t) \prod_{i=1}^{m}\left[\prod_{j=1}^{k_{i}} P\left(g^{j}(t)\right)\right]^{\alpha_{i}}=A \leq \frac{K^{K}}{(K+1)^{K+1}}
$$

and

$$
\limsup _{t \rightarrow \infty}\left(\bar{\lambda}^{-K+1} p(g(t))\right) Q(t) \prod_{i=1}^{m}\left[\prod_{j=2}^{k_{i}} P\left(g^{j}(t)\right)\right]^{\alpha_{i}}+\bar{\lambda}^{-K} Q(g(t)) \prod_{i}^{m}\left[\prod_{j=2}^{k_{i}} P\left(g^{j}(t)\right)\right]^{\alpha_{i}}>1
$$

here $\bar{\lambda}$ is the only real roof of the equation $\lambda-1+A \lambda^{-K}=0$ in $\left[(k+1)^{\frac{1}{K}} A^{\frac{1}{K}, 1}\right]$, then all the solutions of the equation (1.15) oscillated.

Lin Quan-wen, Wu Ying-zhu and Liao Si-quan also studied the equation (1.15) in [6], they had proved:

Theorem 1.6 Supposed:

$$
\liminf _{t \rightarrow \infty} Q(t) \prod_{i=1}^{m}\left(\prod_{j=1}^{k_{i}} P\left(g^{j}(t)\right)\right)^{\alpha_{i}}=A, 0 \leq A \leq \frac{K^{K}}{(K+1)^{K+1}}
$$

and

$$
\limsup _{t \rightarrow \infty} Q(t) \prod_{i=1}^{m}\left(\prod_{j=1}^{k_{i}} P\left(g^{j}(t)\right)\right)^{\alpha_{i}}>\frac{1}{[\lambda(A)]^{k}}
$$

then all the solutions of the equation (1.15) oscillated and reached the corollary 1.1 .

This paper is inspired by [4] and [6], we have studied the oscillation of the equation (1.1), we used the method of studying the solution oscillation of the functional equation with variable coefficients then got some new oscillation rules and applied them to the difference equations and educed some main results. Obviously, when $M=1$, the equation (2.2) change to the equation (1.14). So our results will improve or expand the results of [1],[2] and [4],[6].

\section{Main results}

Let $\sum_{i=1}^{m} \alpha_{i} k_{i}=K$, before we narrate the main results, we quote two lemmas of

[3]: Lemma 1 suppose $0 \leq A \leq \frac{K^{K}}{(K+1)^{K+1}}$

then the equation

$$
A \lambda^{K+1}-\lambda+1=0
$$

has only real root.

Lemma 2 Let $0 \leq A \leq \frac{K^{K}}{(K+1)^{K+1}}$, and defining sequence $\left\{\lambda_{n}\right\}_{n=0}^{\infty}$ as follows

$$
\lambda_{0}=1, \lambda_{n+1}=\left(1-A \lambda_{n}^{K}\right)^{-1}, n=0,1,2, \cdots
$$

then $1 \leq \lambda_{n} \leq \lambda(A), n=0,1,2, \cdots$ and $\lim _{n \rightarrow \infty} \lambda_{n}=\lambda(A)$.

The main results of this paper are as follows:

Theorem 2.1 if one of the following condition come into existence

$$
\text { 1) } \begin{aligned}
\liminf _{t \rightarrow \infty} Q(t) \prod_{i=1}^{m}\left(\prod_{j=1}^{k_{i}+M-1} P\left(g^{j}(t)\right)\right)^{\alpha_{i}}=A>\frac{K^{K}}{(K+1)^{K+1}} \\
2) 0 \leq A \leq \frac{K^{K}}{(K+1)^{K+1}}
\end{aligned}
$$

and

$$
\limsup _{t \rightarrow \infty} Q(t) \prod_{i=1}^{m}\left(\prod_{j=1}^{k_{i}+M-1} P\left(g^{j}(t)\right)\right)^{\alpha_{i}}>\frac{1}{[\lambda(A)]^{k}}
$$

Where $\lambda(A)$ is the only one real roof of equation $(2.2)$ in $\left[1,((K+1) A)^{-\frac{1}{K}}\right]$. Then all the solutions of the equation $(1.1)$ oscillate. 
Proof: suppose equation (1.1) had a nonoscillatory solution $x(t)$, and $x(t)>0, t \in I, t \in t_{1} \in I$ because $\liminf _{t \rightarrow \infty} g(t)=\infty$, then exist $t_{2} \in I$ and $t_{2}>t_{1}$, Marking $x\left(g^{k_{i}+K(t)}\right)>0, t \in \in I, t \geq t_{2}, i=1,2, \cdots m$ Thus from equation (1.1) we have $x(g(t)) \geq P(t) x(t)$, By the iteration, we obtain

$$
x\left(g^{K_{i}+M}(t)\right) \geq \prod_{j=1}^{k_{i}+M-1} P\left(g^{j}(t)\right) x(g(t)), i=1,2, \cdots, m
$$

We divide it into two cases:

(a)Let $A \geq \frac{K^{K}}{(K+1)^{K+1}}$ marking $\beta=\frac{(K+1)^{K+1}}{K^{K}}$.

Substituting (2.5) into (1.1), we obtain

$$
x(g(t))=P(t) x(t)+Q(t) \prod_{i=1}^{m}\left(\prod_{j=1}^{k_{i}+M-1} p\left(g^{j}(t)\right) x(g(t))\right)^{\alpha_{i}} \geq P(t) x(t)+A x(g(t))
$$

We have $A \leq 1$ and

$$
\frac{p(t) x(t)}{x(g(t))} \leq 1-A=\frac{1}{A^{\frac{1}{K}}}\left[A^{f r a c 1 K}(1-A)\right] \leq A^{\frac{1}{K}} \max _{0 \leq \delta \leq 1}\left\{\delta^{\frac{1}{K}}(1-\delta)\right\}=\frac{1}{A^{\frac{1}{K}}} \frac{K}{(K+1)^{1+\frac{1}{K}}}=\frac{1}{(\beta A)^{\frac{1}{K}}}
$$

And

$$
x(g(t)) \geq(\beta A)^{\frac{1}{K}} p(t) x(t)
$$

from the iteration, we obtain

$$
x\left(g^{k_{i}+M}(t)\right) \geq \beta A \prod_{j=1}^{k_{i}+M-1} p\left(g^{j}(t)\right) x(g(t))
$$

Substituting (2.6) into (1.1), we have

$$
x(g(t))=P(t) x(t)+(\beta A) Q(t) \prod_{i=1}^{m}\left(\prod_{j=1}^{K_{i}+M-1} p(g(t))\right)^{\alpha_{i}} x(g(t)) \geq p(t) x(t)+\left(\beta A^{2}\right) x(g(t))
$$

So we obtain

$$
\begin{gathered}
\frac{p(t) x(t)}{x(g(t))} \leq 1-\beta A^{2}=\frac{1}{\left(\beta A^{2}\right)^{\frac{1}{K}}}\left[\left(\beta A^{2}\right)^{\frac{1}{K}}\left(1-\beta A^{2}\right)\right]=\frac{1}{\left(\beta A^{2}\right)^{\frac{1}{K}}} \max _{0 \leq \delta \leq 1}\left\{\delta^{\frac{1}{K}}(1-\delta)\right\} \\
=\frac{1}{\left(\beta A^{2}\right)^{\frac{1}{K}}} \frac{1}{\beta^{\frac{1}{K}}}=\frac{1}{\left(\beta A^{2}\right)^{\frac{1}{K}}} \frac{K}{(K+1)^{1+\frac{1}{K}}}=\frac{1}{\left(\beta^{2} A^{2}\right)^{\frac{1}{K}}}
\end{gathered}
$$

That is $x(g(t)) \geq\left(\beta^{2} A^{2}\right)^{\frac{1}{K}} p(t) x(t)$.

By iterating it again, we obtain

$$
x\left(g^{k_{i}+M}(t)\right) \geq(\beta A)^{2} \prod_{i=1}^{m}\left[\prod_{j=1}^{K_{i}+M-1} p\left(g^{j}(t)\right)\right] x(g(t)), i=1,2, \cdots m
$$

By repeating the process, in any $n=1,2,3, \cdots$ we have

$$
x\left(g^{k_{i}+M}(t)\right) \geq(\beta A)^{n} \prod_{i=1}^{m}\left[\prod_{j=1}^{K_{i}+M-1} p\left(g^{j}(t)\right)\right] x(g(t))
$$

Because $\beta A>1$, so there must be an $n^{*}$, let the equation (2.11) come into existence. And

$$
(\beta A)^{n^{*}+1}>\beta
$$

Substituting (2.7) into (1.1), we obtain:

$$
x(g(t)) \geq P(t) x(t)+(\beta A)^{n} Q(t) \prod_{i=1}^{m}\left[\prod_{j=1}^{K_{i}+M-1} p\left(g^{j}(t)\right)\right]^{\alpha_{i}} x(g(t)) \geq(\beta A)^{n} A x(g(t))
$$

That is

$$
1 \geq(\beta A)^{n} A \text { or } \beta \geq(\beta A)^{n+1}
$$


And we can see that it conflict with (2.8).

(b) $0 \leq A \leq \frac{K^{k}}{(K+1)^{K+1}}$

Substituting (2.5) into (1.1), we obtain:

$$
x(g(t)) \geq P(t) x(t)+Q(t) \prod_{i=1}^{m}\left[\prod_{j=1}^{K_{i}+M-1} p\left(g^{j}(t)\right)\right]^{\alpha_{i}} x(g(t)) \geq p(t) x(t)+A x(g(t))=p(t) x(t)+\lambda_{0}^{K} A x(g(t))
$$

here $\lambda_{0}=1$.

Then $x(g(t)) \geq\left(1-\lambda_{0}^{K} A\right)^{-1} P(t) x(t)$,ordering $\lambda_{1}=\left(1-\lambda_{0}^{K} A\right)^{-1}$.

So $x(g(t)) \geq \lambda_{1} P(t) x(t)$.

Next, we are going to prove:

$$
x(g(t)) \geq \lambda_{n} P(t) x(t), n=1,2, \cdots
$$

Suppose $x(g(t)) \geq \lambda_{1} P(t) x(t), i \geq 1$, from the iteration, we obtain:

$$
\left.x\left(g^{k_{i}+M}(t)\right) \geq \lambda_{i}^{k_{i}} \prod_{i=1}^{m} p\left(g^{j}(t)\right)\right] x(g(t))
$$

Substituting (2.11) into (1.1), we will have:

$$
x(g(t)) \geq P(t) x(t)+\lambda_{i}^{\sum \alpha_{i} k_{i}} Q(t) \prod_{i=1}^{m}\left[\prod_{j=1}^{K_{i}+M-1} p\left(g^{j}(t)\right)\right]^{\alpha_{i}} x(g(t)) \geq p(t) x(t)+\lambda_{i}^{K} A x(g(t))
$$

Then $x(g(t)) \geq\left(1-\lambda_{1}^{K} A\right)^{-1} P(t) x(t)=\lambda_{i+1} P(t) x(t)$.

So, from the mathematical induction method, we know that (2.10) exists, from the iteration, we obtain:

$$
x\left(g^{k_{i}+M}(t)\right) \geq \lambda_{n}^{k_{i}} \prod_{j=1}^{K_{i}+M-1} p\left(g^{j}(t)\right) x(g(t))
$$

Substituting the (2.12) into (1.1), we reach

$$
x(g(t)) \geq \lambda_{n}^{K_{i}} Q(t) \prod_{i=1}^{m}\left[\prod_{j=1}^{K_{i}+M-1} p\left(g^{j}(t)\right)\right]^{\alpha_{i}} x(g(t))
$$

or

$$
Q(t) \prod_{i=1}^{m}\left[\prod_{j=1}^{K_{i}+M-1} p\left(g^{j}(t)\right)\right]^{\alpha_{i}} \leq \frac{1}{\lambda_{n}^{K}}
$$

This contains:

$$
\limsup _{t \rightarrow \infty} Q(t) \prod_{i=1}^{m}\left[\prod_{j=1}^{K_{i}+M-1} p\left(g^{j}(t)\right)\right]^{\alpha_{i}} \leq \frac{1}{\lambda_{n}^{K}}
$$

Let $t \rightarrow \infty$ and get the superior limit of (2.13), from the lemma 2, we obtain:

$$
\limsup _{t \rightarrow \infty} Q(t) \prod_{i=1}^{m}\left[\prod_{j=1}^{K_{i}+M-1} p\left(g^{j}(t)\right)\right]^{\alpha_{i}} \leq \frac{1}{\lambda_{n}^{K}(A)}
$$

And this contradicts with what we had known before. The lemma is proved completely.

When $m=1, K_{i}=1, K=1$,from the theorem, we can obtain:

Corollary 1: if one of the following condition is true,

1) $\liminf _{t \rightarrow \infty} Q(t) p(g(t))=A>\frac{1}{4}$

2) $0 \leq A \leq \frac{1}{4}$, and $\lim \sup Q(t) p(g(t))>\frac{1+\sqrt{1-4 A}}{2}$

And this is the result of [1]. 


\section{Applications}

Because functional equation (1.1) includes difference equation as its special condition with discrete arguments and continuous arguments So if we let $g(t)=\pi+t, t \in R^{+}, I=R^{+}$The equation (1.1) becomes into a difference equation with the continuous arguments of the form:

$$
x(\pi+t)=P(t) x(t)+Q(t) \prod_{i=1}^{m}\left|x\left(t+\left(k_{i}+M\right) \pi\right)\right|^{\alpha_{i}} \operatorname{sign} x\left(t+\left(k_{i}+M\right) \pi\right)
$$

From the theorem 2.1, we can obtain:

Theorem 3.1 If one of the following condition is true,

1) $\liminf _{t \rightarrow \infty} Q(t) \prod_{i=1}^{m}\left(\prod_{j=1}^{k_{i}+M-1} P(t+j \pi)\right)^{\alpha_{i}}=A>\frac{K^{K}}{(K+1)^{K+1}}$

2) $0 \leq A \leq \frac{K^{K}}{(K+1)^{K+1}}$, and $\limsup _{t \rightarrow \infty} Q(t) \prod_{i=1}^{m}\left(\prod_{j=1}^{k_{i}+M-1} P(t+j \pi)\right)^{\alpha_{i}}>\frac{1}{[\lambda(A)]^{K}}$

Then all the solutions of the equation (3.1) is oscillatory.

\section{References}

Golda, W and Werbowski, J. (1994). Oscillation of Linear Functional Equations of the Second Order. Funkcialaj Ekvacioj, 37(2): 211-228.

Lin, Q. W, WU, Y. Z. and Liao, S. Q. (2007). Oscillation Criferia of Nonlinear Functional Equations with Variable Coefficient. J.MaoMing university, (17): 64-66.

Nowakowska, W, Werbowski, J. (1995). Oscillation of Linear Functional Equations of Higher Order. Arch. Math, 31: 251-258.

Nowakowska, W, Werbowski, J. (2001). Oscillation Behavior of Solutions of Functional Equations. Nonlinear Anaysis, (44): 756-775.

Zhou, Yong and Yu, Yuanhong. (1999). The Oscillation of Functional Equations with Variable Coefficients. J.sys.Sci.Math. Scis. 19(3): 348-352.

Zhou, Yong, Liu, Zhenghong and Yu, Yuanhong. (2000). Oscillation Criteria of Functional Equations with Variable Coefficients. Act. Mathematica Appl.smica, 23(3): 413-419. 